\title{
Numerical Analysis of the Transmission Model of Dengue on SEIR Model
}

\author{
Rattiya Sungchasit ${ }^{1 *}$, Puntani Pongsumpun ${ }^{2}$ \\ ${ }^{1}$ Department of Mathematics, Faculty of Science and Technology, Phuket Rajabhat University \\ Muang District, Phuket, 83000, Thailand. \\ 2 Department of Mathematics, Faculty of Science, King Mongkut's institute of Technology Ladkrabang, \\ Chalongkrung road Ladkrabang, Bangkok 10520, Thailand.
}

* Corresponding author. Email: tan_sungchasit@hotmail.com

Manuscript submitted December 1, 2016; accepted February 13, 2017.

doi: 10.17706/ijbbb.2017.7.2.93-101

\begin{abstract}
In this paper, we used SEIR model to describe the transmission of dengue disease in mosquito due to the different dengue transmission rate in each season. Aedes aegypti and Aedes albopictus are primary vectors for the disease. The human population is separated into three population groups such as human in rainy season, human in winter season, human in summer season and vector population. We use standard dynamical analysis method for analyzing mathematical model. The basic reproductive number of disease is found. The stability conditions of the disease free equilibrium state for $R_{0}<1$ and the endemic state are determined for $R_{0}>1$.
\end{abstract}

Key words: Transmission model, mathematical model, SEIR model.

\section{Introduction}

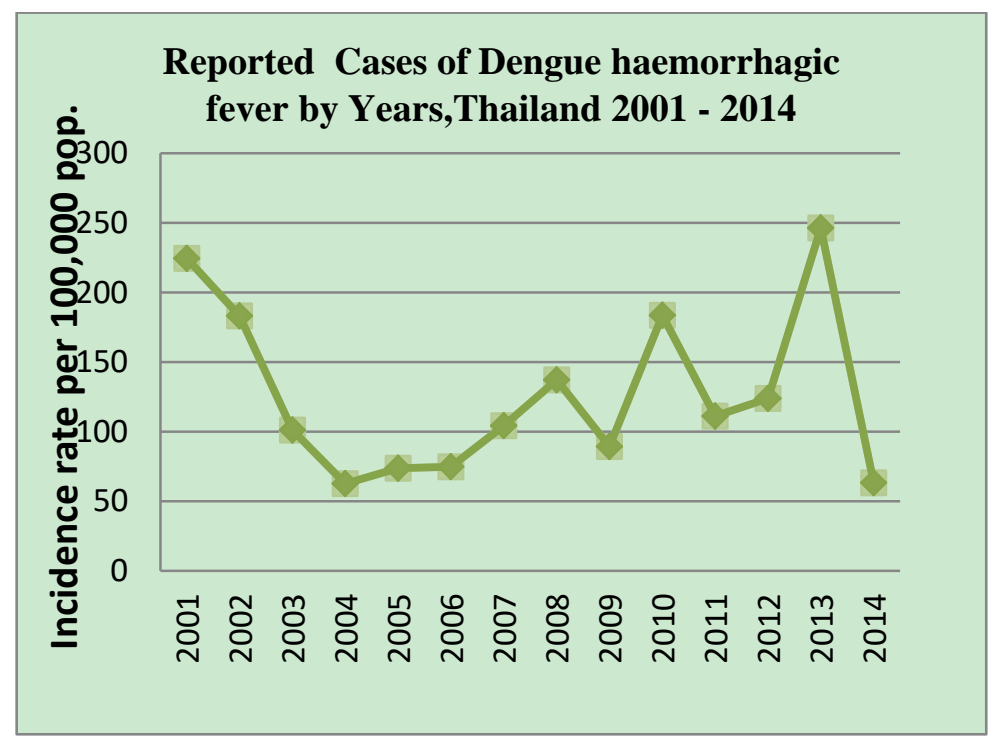

Fig. 1. The reports of cases rates of dengue disease in Thailand by year during 2001-2014 [1].

Dengue fever is the arbovirus infectious disease which can be found in tropical region and subtropical 
regions of the world. It is estimated that around two - thirds of the world's population and approximately 50 - 100 million cases occur each year [2], [3]. It is transmitted to human beings by a mosquito belonging to the Genus Aedes. There are four serotypes of dengue virus, namely DEN-1, DEN-2, DEN-3 and DEN-4. Dengue fever is transmitted to human by biting of the female mosquitoes infected with a dengue virus. When the female mosquitoes bite the human with dengue virus in their blood, thus the human are exposed and infected. It can't be spread directly from human to another human. In a human, the virus incubates for 3 to 14 days before symptoms appear, with an average symptom onset at 4 to 7 days. The exactly symptoms depend on age. In older children, teenage and adults, the most common symptoms of dengue disease are a fever that comes on quickly and lasts 2 to 7 days, a headache, muscle and joint pain (dengue fever is also known as "breakbone fever"), a red rash that starts on chest, pain behind eyes and feeling sick and vomiting [3]-[9]. Dengue fever is caused by a type of virus called a flavivirus, which is transmitted by infected female Aedes mosquitoes. The two known species that can transmit dengue virus are Aedes Aegypti and Aedes Albopictus. The former is Anthropophilic and thrives in heavily populated cities, biting primarily during the day; the latter, meanwhile, inhabits rural areas.

In order to have a more comprehensive knowledge about these diseases and for the preparation of strategies, mathematical modeling becomes an important tool. Mathematical models were developed to investigate the infectious epidemiology. The moisture content, temperature, season and rainfall are influence to the mosquito development. Dengue infection is endemic in Thailand. From the data of Dengue cases rate and deaths rate in 2001-2014, we can see that most dengue patients are occurred season, as we can see in Fig. 1.

Esteva and Vargas proposed their mathematical model for the transmission of dengue virus infection in a constant human population. They supposed that the population can be infected only one time [3]. This disease is occurred by biting of infected Aedes aegypti and Aedes albopictus mosquitoes. Dengue outbreak is found during the season [4]-[7]. Each Aedes mosquito has the different dengue outbreaks and they are depend on the temperature of the environment and areas [4]-[10].

In this study, we develop the transmission of dengue disease by formulating the mathematical models. We used SEIR model for analyzing and finding the method to decrease the outbreak of this disease. We analyze dengue model of seasonality compartment (rainy season, winter season and summer season).

\section{Methodology}

The model is based on the standard SEIR model, In this study, we assume that the total human and mosquito population have constant sizes [3]. The human population is divided into susceptible, exposed, infected and recovered classes for the first model. The mosquito population is divided into susceptible and infected classes because the mosquito never recover from infection. The model considers transmission of dengue virus in human and mosquito populations:

The dynamics of population are given human by

$$
\begin{gathered}
\frac{d S}{d t}=\theta N_{t}-\frac{\beta_{r} P_{r}}{N_{t}} S I_{v}-\frac{\beta_{w} P_{w}}{N_{t}} S I_{v}-\frac{\beta_{s} P_{s}}{N_{t}} S I_{v}-\tau_{d} S \\
\frac{d E_{r}}{d t}=\frac{\beta_{r} P_{r}}{N_{t}} S I_{v}-\tau_{d} E_{r}-\alpha_{r} E_{r} \\
\frac{d E_{w}}{d t}=\frac{\beta_{w} P_{w}}{N_{t}} S I_{v}-\tau_{d} E_{w}-\alpha_{s} E_{w}
\end{gathered}
$$




$$
\begin{aligned}
\frac{d E_{s}}{d t} & =\frac{\beta_{s} P_{s}}{N_{t}} S I_{v}-\tau_{d} E_{s}-\alpha_{s} E_{s} \\
\frac{d E_{r}}{d t} & =\frac{\beta_{r} P_{r}}{N_{t}} S I_{v}-\tau_{d} E_{r}-\alpha_{r} E_{r} \\
\frac{d I_{r}}{d t} & =\alpha_{r} E_{r}-\gamma_{r} I_{r}-\tau_{d} I_{r} \\
\frac{d I_{w}}{d t} & =\alpha_{w} E_{w}-\gamma_{w} I_{w}-\tau_{d} I_{w} \\
\frac{d I_{s}}{d t} & =\alpha_{s} E_{s}-\gamma_{s} I_{s}-\tau_{d} I_{s} \\
\frac{d R}{d t} & =\gamma_{r} I_{r}+\gamma_{r} I_{r}+\gamma_{s} I_{s}-\tau_{d} I_{s}
\end{aligned}
$$

We defined variables as follows: $S$ is the number of susceptible human population. $E_{r}, E_{w}$ and $E_{s}$ are the number of exposed human population in rainy season, winter season and summer season. $I_{r}, I_{w}$ and $I_{s}$ are the number of infectious human population in rainy season, winter season and summer season. $R$ is the number of recovered human population.

The dynamics of the mosquito population are given by:

$$
\begin{gathered}
\frac{d S_{v}}{d t}=C-\frac{P_{v}}{N_{t}} S_{v}\left(I_{r}+I_{w}+I_{s}\right)-\tau_{v} S_{v} \\
\frac{d I_{v}}{d t}=\frac{P_{v}}{N_{t}} S_{v}\left(I_{r}+I_{w}+I_{s}\right)-\tau_{v} I_{v}
\end{gathered}
$$

We define: $S_{v}$ is the number of susceptible mosquito is the number of population and $I_{v}$ is the number of infectious mosquito population

We suppose that $N_{t}=S+E_{r}+E_{w}+E_{s}+I_{r}+I_{w}+I_{s}+R$ and $N_{v}=S_{v}+I_{v}$.

From the above conditions, we can have $\frac{d N_{t}}{d t}=0$ and $\frac{d N_{v}}{d t}=0$.

The total human and mosquito populations have constant sizes. Thus rates of change for human and mosquito populations equal to zero. Thus, the birth and death rates are equivalent for human populations.

We introduce the new variables:

$$
S^{\prime}=\frac{S}{N_{t}}, E_{r}^{\prime}=\frac{E_{r}}{N_{t}}, E_{w}{ }^{\prime}=\frac{E_{w}}{N_{t}}, E_{s}{ }^{\prime}=\frac{E_{s}}{N_{t}}, I_{r}{ }^{\prime}=\frac{I_{r}}{N_{t}}, I_{w}{ }^{\prime}=\frac{I_{w}}{N_{t}}, I_{s}{ }^{\prime}=\frac{I_{s}}{N_{t}} R^{\prime}=\frac{R}{N_{t}}, S_{v}{ }^{\prime}=\frac{S_{v}}{N_{v}}, I_{v}{ }^{\prime}=\frac{I_{v}}{N_{v}} .
$$

The reduced equations become:

$$
\frac{d S^{\prime}}{d t}=\tau_{d}-\frac{\beta_{r} P_{r}}{N_{t}} S^{\prime} I_{v}^{\prime} N_{v}-\frac{\beta_{w} P_{w}}{N_{t}} S^{\prime} I_{v}^{\prime} N_{v}-\frac{\beta_{s} P_{s}}{N_{t}} S^{\prime} I_{v}^{\prime} N_{v}-\tau_{d} S^{\prime}
$$




$$
\begin{aligned}
& \frac{d E_{r}{ }^{\prime}}{d t}=\frac{\beta_{r} P_{r}}{N_{t}} S^{\prime} I_{v}{ }^{\prime} N_{v}-\tau_{d} E_{r}^{\prime}-\alpha_{r} E_{r}^{\prime} \\
& \frac{d E_{w}{ }^{\prime}}{d t}=\frac{\beta_{w} P_{w}}{N_{t}} S^{\prime} I_{v}^{\prime} N_{v}-\tau_{d} E_{w}{ }^{\prime}-\alpha_{w} E_{w}{ }^{\prime} \\
& \frac{d E_{s}{ }^{\prime}}{d t}=\frac{\beta_{s} P_{s}}{N_{t}} S^{\prime} I_{v}{ }^{\prime} N_{v}-\tau_{d} E_{s}{ }^{\prime}-\alpha_{s} E_{s}{ }^{\prime} \\
& \frac{d I_{r}^{\prime}}{d t}=\alpha_{r} E_{r}^{\prime}-\gamma_{r} I_{r}^{\prime}-\tau_{d} I_{r}^{\prime} \\
& \frac{d I_{w}^{\prime}}{d t}=\alpha_{w} E_{w}{ }^{\prime}-\gamma_{w} I_{w}^{\prime}-\tau_{d} I_{w}^{\prime} \\
& \frac{d I_{s}^{\prime}}{d t}=\alpha_{s} E_{s}^{\prime}-\gamma_{s} I_{s}^{\prime}-\tau_{d} I_{s}^{\prime} \\
& \frac{d I_{v}{ }^{\prime}}{d t}=\frac{P_{v}}{N_{t}}\left(1-I_{v}{ }^{\prime}\right)\left(I_{r}{ }^{\prime} N_{t}+I_{w}{ }^{\prime} N_{t}+I_{s}{ }^{\prime} N_{t}\right)-\tau_{v} I_{v}{ }^{\prime}
\end{aligned}
$$

With the conditions $S+E_{r}+E_{w}+E_{s}+I_{r}+I_{w}+I_{s}+R=1$ and $S_{v}+I_{v}=1$.

Table 1. Definitions of Variables and Parameters for Our Model

\begin{tabular}{|c|l|}
\hline $\begin{array}{l}\text { Variable/ } \\
\text { parameter }\end{array}$ & definition \\
\hline$N_{t}$ & Total human population \\
\hline$N_{v}$ & Total mosquito population \\
\hline$\tau_{d}$ & The death rate of human population \\
\hline$\tau_{v}$ & The death rate of mosquito population \\
\hline$\theta$ & The birth rate of human population \\
\hline$P_{r}$ & $\begin{array}{l}\text { The transmission probability of dengue disease from mosquito to } \\
\text { human in rainy season }\end{array}$ \\
\hline$P_{w}$ & $\begin{array}{l}\text { The transmission probability of dengue disease from mosquito to } \\
\text { human in winter season }\end{array}$ \\
\hline$P_{s}$ & $\begin{array}{l}\text { The transmission probability of dengue disease from mosquito to } \\
\text { human in summer season }\end{array}$ \\
\hline$P_{v}$ & $\begin{array}{l}\text { The transmission probability of dengue disease from mosquito to human } \\
\text { in summer season }\end{array}$ \\
\hline$\beta_{r}$ & The biting rate of mosquito population in rainy season \\
\hline$\beta_{w}$ & The biting rate of mosquito population in winter season \\
\hline$\beta_{s}$ & The biting rate of mosquito population in summer season \\
\hline$\alpha_{s}$ & The incubation rate of human population in rainy season \\
\hline$\alpha_{w}$ & The incubation rate of human population in winter season \\
\hline$\alpha_{s}$ & The incubation rate of human population in summer season \\
\hline$\gamma_{r}$ & The recovery rate of human population in rainy season \\
\hline$\gamma_{w}$ & The recovery rate of human population in winter season \\
\hline$\gamma_{s}$ & The recovery rate of human population in summer season \\
\hline
\end{tabular}




\subsection{Analysis of the Mathematical Model}

From the above equations, after setting (12)-(19) to zero. We find two equilibrium states:

i) The disease free equilibrium state $F_{0}=(1,0,0,0,0,0,0,0)$

ii) The endemic disease equilibrium state

$$
F_{1}=\left(S^{*}, E_{r}^{*}, E_{w}^{*}, E_{s}^{*}, I_{r}{ }^{*}, I_{w}{ }^{*}, I_{s}{ }^{*}, I_{v}^{*}\right)
$$

where

$$
\begin{aligned}
& S^{*}=\frac{N_{t} \tau_{d}}{I_{v}{ }^{*} N_{v}\left(P_{r} \beta_{r}+\beta_{w} P_{w}+\beta_{s} P_{s}\right)+N_{t} \tau_{d}} \\
& E_{r}^{*}=\frac{I_{v}^{*} N_{v} P_{r} \beta_{r} \tau_{d}}{\left(\alpha_{r}+\tau_{d}\right)\left(I_{v}^{*} N_{v}\left(P_{r} \beta_{r}+\beta_{w} P_{w}+\beta_{s} P_{s}\right)+N_{t} \tau_{d}\right)} \\
& E_{w}^{*}=\frac{I_{v}{ }^{*} N_{v} P_{w} \beta_{w} \tau_{d}}{\left(\alpha_{w}+\tau_{d}\right)\left(I_{v}^{*} N_{v}\left(P_{r} \beta_{r}+\beta_{w} P_{w}+\beta_{s} P_{s}\right)+N_{t} \tau_{d}\right)} \\
& E_{s}^{*}=\frac{I_{v}^{*} N_{v} P_{s} \beta_{s} \tau_{d}}{\left(\alpha_{s}+\tau_{d}\right)\left(I_{v}{ }^{*} N_{v}\left(P_{r} \beta_{r}+\beta_{w} P_{w}+\beta_{s} P_{s}\right)+N_{t} \tau_{d}\right)} \\
& I_{r}^{*}=\frac{I_{v}{ }^{*} N_{v} P_{r} \beta_{r} \tau_{d} \alpha_{r}}{\left(\left(\alpha_{r}+\tau_{d}\right)\left(\gamma_{r}+\tau_{d}\right)\left(I_{v}{ }^{*} N_{v}\left(P_{r} \beta_{r}+\beta_{w} P_{w}+\beta_{s} P_{s}\right)+N_{t} \tau_{d}\right)\right)} \\
& I_{w}{ }^{*}=\frac{I_{v}{ }^{*} N_{v} P_{w} \beta_{w} \tau_{d} \alpha_{w}}{\left(\left(\alpha_{w}+\tau_{d}\right)\left(\gamma_{w}+\tau_{d}\right)\left(I_{v}{ }^{*} N_{v}\left(P_{r} \beta_{r}+\beta_{w} P_{w}+\beta_{s} P_{s}\right)+N_{t} \tau_{d}\right)\right)} \\
& I_{s}^{*}=\frac{I_{v}^{*} N_{v} P_{s} \beta_{s} \tau_{d} \alpha_{s}}{\left(\left(\alpha_{s}+\tau_{d}\right)\left(\gamma_{s}+\tau_{d}\right)\left(I_{v}^{*} N_{v}\left(P_{r} \beta_{r}+\beta_{w} P_{w}+\beta_{s} P_{s}\right)+N_{t} \tau_{d}\right)\right)} \\
& I_{v}^{*}=\left[\tau _ { d } \left(-N_{v} P_{v}\left(P_{w} \alpha_{w} \beta_{w}\left(\alpha_{r}+\tau_{d}\right)\left(\alpha_{s}+\tau_{d}\right)\left(\gamma_{r}+\tau_{d}\right)\left(\gamma_{s}+\tau_{d}\right)+\left(\alpha_{w}+\tau_{d}\right)\left(\gamma_{w}+\tau_{d}\right)\right.\right.\right. \\
& \left.\left(P_{s} \alpha_{s} \beta_{s}\left(\alpha_{r}+\tau_{d}\right)\left(\gamma_{r}+\tau_{d}\right)+P_{r} \alpha_{r} \beta_{r}\left(\alpha_{s}+\tau_{d}\right)\left(\gamma_{s}+\tau_{d}\right)\right)\right)+N_{t}\left(\alpha_{r}+\tau_{d}\right)\left(\alpha_{s}+\tau_{d}\right) \\
& \left.\left(\alpha_{w}+\tau_{d}\right)\left(\gamma_{r}+\tau_{d}\right)\left(\gamma_{s}+\tau_{d}\right)\left(\gamma_{w}+\tau_{d}\right) \tau_{v}\right] /\left[-\left\{N _ { v } \left(P _ { v } \tau _ { d } \left(P_{w} \alpha_{w} \beta_{w}\left(\alpha_{r}+\tau_{d}\right)\left(\alpha_{s}+\tau_{d}\right)\right.\right.\right.\right. \\
& \left(\gamma_{r}+\tau_{d}\right)\left(\gamma_{s}+\tau_{d}\right)+\left(\alpha_{w}+\tau_{d}\right)\left(\gamma_{w}+\tau_{d}\right)\left(P_{s} \alpha_{s} \beta_{s}\left(\alpha_{r}+\tau_{d}\right)\left(\gamma_{r}+\tau_{d}\right)+\right. \\
& \left.\left.P_{r} \alpha_{r} \beta_{r}\left(\alpha_{s}+\tau_{d}\right)\left(\gamma_{s}+\tau_{d}\right)\right)\right)+\left(P_{r} \beta_{r}+P_{s} \beta_{s}+P_{w} \beta_{w}\right)\left(\alpha_{r}+\tau_{d}\right)\left(\alpha_{s}+\tau_{d}\right)\left(\alpha_{w}+\tau_{d}\right) \\
& \left.\left.\left(\gamma_{r}+\tau_{d}\right)\left(\gamma_{s}+\tau_{d}\right)\left(\gamma_{w}+\tau_{d}\right) \tau_{v}\right\}\right]
\end{aligned}
$$

To determine the local stability from equations (12) - (19), we check the sign of eigenvalues. The eigenvalues are the solutions of characteristic equations: $\operatorname{det}(J-\lambda I)=0$; where $\mathrm{J}$ is the jacobian matrix, $\mathrm{I}$ is the identity matrix. If all eigenvalues have negative real parts then the equilibrium state is local stability.

For disease free equilibrium state: Jacobian matrix is defined by 


$$
F_{0}=\left(\begin{array}{cccccccc}
-\tau_{d} & 0 & 0 & 0 & 0 & 0 & 0 & -\left(\frac{\beta_{r} P_{r}}{N_{t}}+\frac{\beta_{w} P_{w}}{N_{t}}+\frac{\beta_{s} P_{s}}{N_{t}}\right) N_{v} \\
0 & -\tau_{d}-\alpha_{r} & 0 & 0 & 0 & 0 & 0 & \frac{\beta_{r} P_{r}}{N_{t}} N_{v} \\
0 & 0 & -\tau_{d}-\alpha_{w} & 0 & 0 & 0 & 0 & \frac{\beta_{w} P_{w}}{N_{t}} N_{v} \\
0 & 0 & 0 & -\tau_{d}-\alpha_{s} & 0 & 0 & 0 & \frac{\beta_{s} P_{s}}{N_{t}} N_{v} \\
0 & \alpha_{r} & 0 & 0 & -\gamma_{r}-\tau_{d} & 0 & 0 & 0 \\
0 & 0 & \alpha_{w} & 0 & 0 & -\gamma_{w}-\tau_{d} & 0 & 0 \\
0 & 0 & 0 & \alpha_{s} & 0 & 0 & -\gamma_{s}-\tau_{d} & 0 \\
0 & 0 & 0 & 0 & P_{v}-\left(-P_{v}\right) & P_{v}-\left(-P_{v}\right) & P_{v}-\left(-P_{v}\right) & \tau_{v}
\end{array}\right)
$$

Jacobian matrix for endemic equilibrium state is given by

$$
F_{1}=\left(\begin{array}{cccccccc}
-\left(\frac{\beta_{r} P_{r}}{N_{t}}+\frac{\beta_{w} P_{w}}{N_{t}}+\frac{\beta_{s} P_{s}}{N_{t}}\right) I_{v}^{\prime} N_{v}-\tau_{d} & 0 & 0 & 0 & 0 & 0 & 0 & -\left(\frac{\beta_{r} P_{r}}{N_{t}}+\frac{\beta_{w} P_{w}}{N_{t}}+\frac{\beta_{s} P_{s}}{N_{t}}\right) S^{\prime} N_{v} \\
\frac{\beta_{r} P_{r}}{N_{t}} I_{v}^{\prime} N_{v} & -\tau_{d}-\alpha_{r} & 0 & 0 & 0 & 0 & 0 & \frac{\beta_{r} P_{r}}{N_{t}} S^{\prime} N_{v} \\
\frac{\beta_{w} P_{w}}{N_{t}} I_{v}^{\prime} N_{v} & 0 & -\tau_{d}-\alpha_{w} & 0 & 0 & 0 & 0 & \frac{\beta_{w} P_{w}}{N_{t}} S^{\prime} N_{v} \\
\frac{\beta_{s} P_{s}}{N_{t}} I_{v}^{\prime} N_{v} & 0 & 0 & -\tau_{d}-\alpha_{s} & 0 & 0 & 0 & \frac{\beta_{s} P_{s}}{N_{t}} S^{\prime} N_{v} \\
0 & \alpha & 0 & 0 & -\gamma_{r}-\tau_{d} & 0 & 0 & 0 \\
0 & 0 & \alpha_{w} & 0 & 0 & -\gamma_{w}-\tau_{d} & 0 & 0 \\
0 & 0 & 0 & \alpha_{s} & 0 & 0 & -\gamma_{s}-\tau_{d} & 0 \\
0 & 0 & 0 & 0 & P_{v}-\left(I_{v}^{\prime}-P_{v}\right) & P_{v}-\left(I_{v}^{\prime}-P_{v}\right) P_{v}-\left(I_{v}^{\prime}-P_{v}\right) & \tau_{v}
\end{array}\right)
$$

We check the sign of all eigenvalues for disease free equilibrium state and endemic equilibrium state, then we conclude that the disease free equilibrium state is local stability for $R_{0}<1$ and endemic equilibrium state is local stability for $R_{0}>1$; where

$$
\begin{aligned}
R_{0}= & {\left[\tau _ { d } \left(-N_{v} P_{v}\left(P_{w} \alpha_{w} \beta_{w}\left(\alpha_{r}+\tau_{d}\right)\left(\alpha_{s}+\tau_{d}\right)\left(\gamma_{r}+\tau_{d}\right)\left(\gamma_{s}+\tau_{d}\right)+\left(\alpha_{w}+\tau_{d}\right)\left(\gamma_{w}+\tau_{d}\right)\right.\right.\right.} \\
& \left.\left.\left(P_{s} \alpha_{s} \beta_{s}\left(\alpha_{r}+\tau_{d}\right)\left(\gamma_{r}+\tau_{d}\right)+P_{r} \alpha_{r} \beta_{r}\left(\alpha_{s}+\tau_{d}\right)\left(\gamma_{s}+\tau_{d}\right)\right)\right)\right] / \\
& {\left[N_{t}\left(\alpha_{r}+\tau_{d}\right)\left(\alpha_{s}+\tau_{d}\right)\left(\alpha_{w}+\tau_{d}\right)\left(\gamma_{w}+\tau_{d}\right)\left(\gamma_{r}+\tau_{d}\right)\left(\gamma_{s}+\tau_{d}\right) \tau_{v}\right] }
\end{aligned}
$$

\section{Numerical Results}

Numerical simulation is presented for the transmission of dengue fever. Data on the number of dengue fever cases in Thailand, we are interested in transmission of dengue disease with the season. The values of the parameter used in this study are as follows: $\tau_{d}=1 /(365 * 76.8)$ per day corresponds to a life expectancy of 76.8 years in human. The other parameters are arbitrarily chosen. We presented the numerical solutions of (12)-(19) for endemic equilibrium state on the follows Fig. 2 and Fig. 3.

The solutions converge to the disease free state $(1,0,0,0,0,0,0,0)$. We can see that the solutions converge to the disease free state.

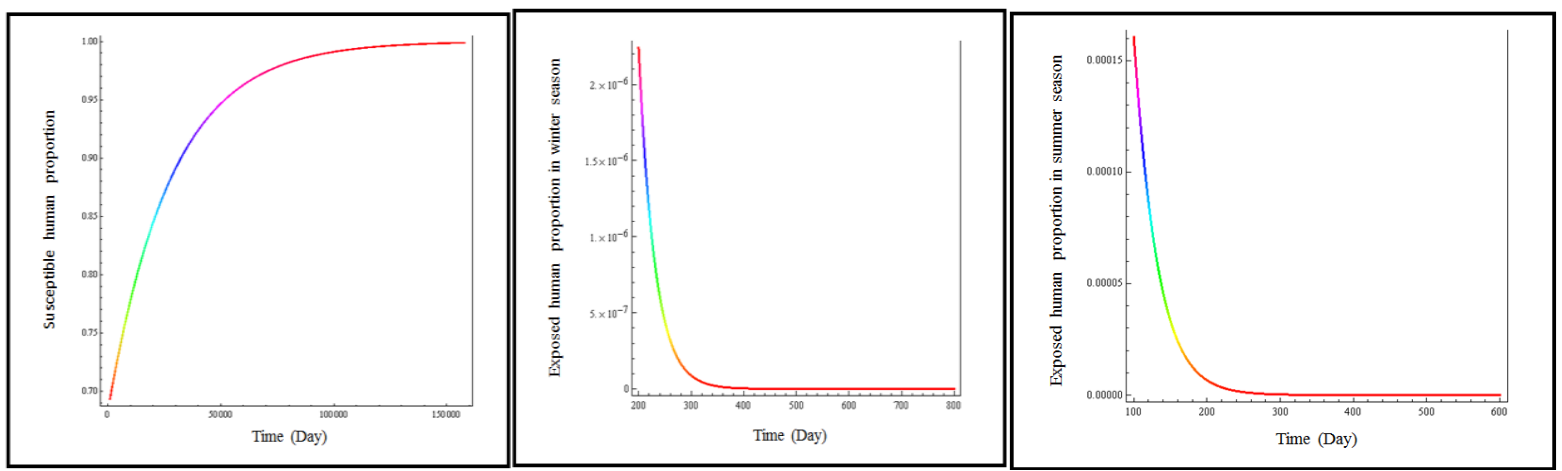




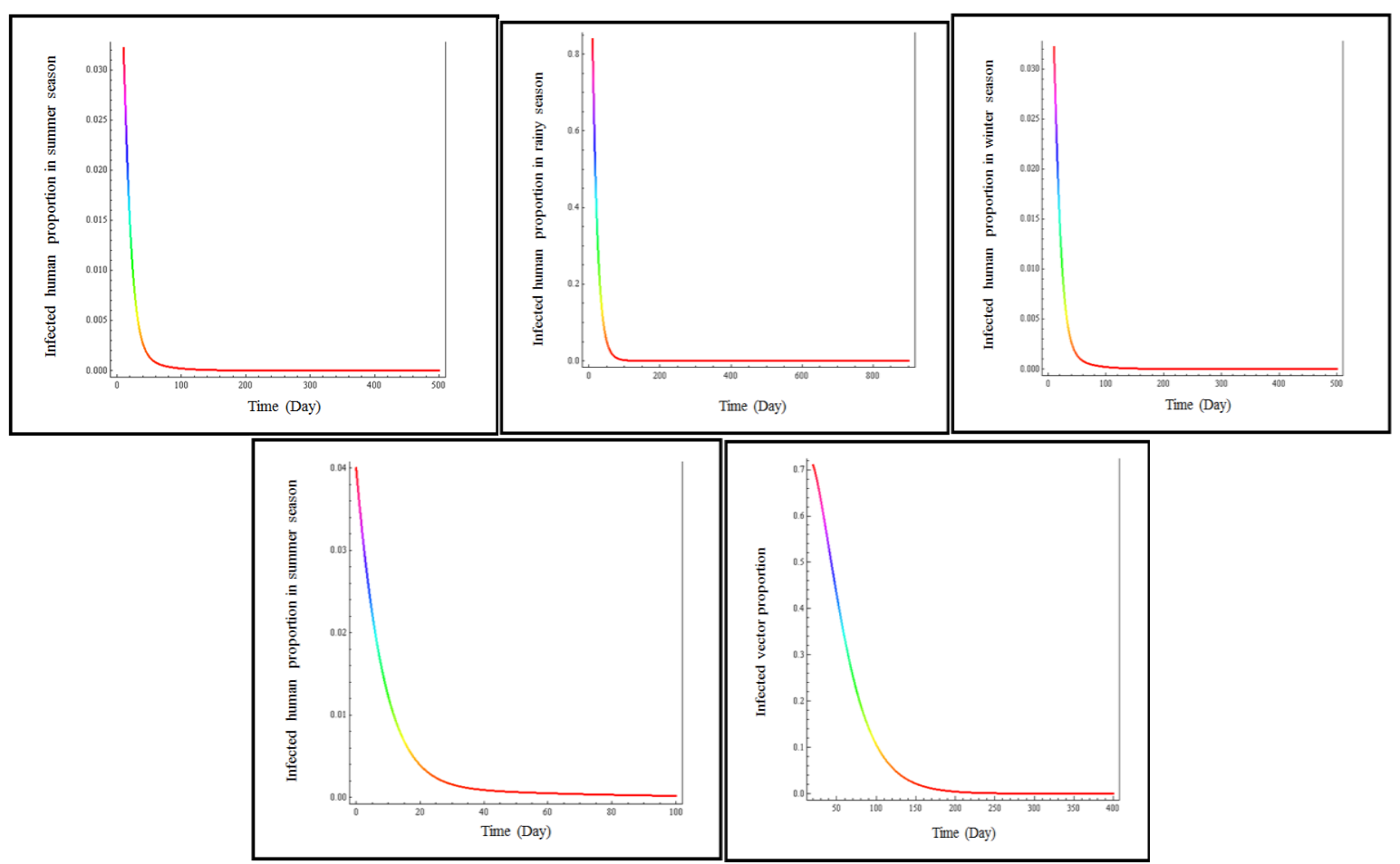

Fig. 2. Time series solutions of $S, E_{r}, E_{w}, E_{s}, I_{r}, I_{w}, I_{s}$ and $I_{v}$ where the parameters are given by $N_{t}=100,000, \tau_{d}=1 /(365 * 76.8), \tau_{v}=1 / 30, \beta_{r}=0.25, \beta_{w}=0.167, \beta_{s}=0.125, P_{r}=0.05, \quad P_{w}=0.10$, $P_{s}=0.20, P_{v}=0.143, \alpha_{r}=0.33, \alpha_{w}=0.25, \alpha_{s}=0.142, \gamma_{r}=0.071, \gamma_{w}=0.10, \gamma_{s}=0.167, N_{v}=1,000$ and $R_{0}=0.021060$.
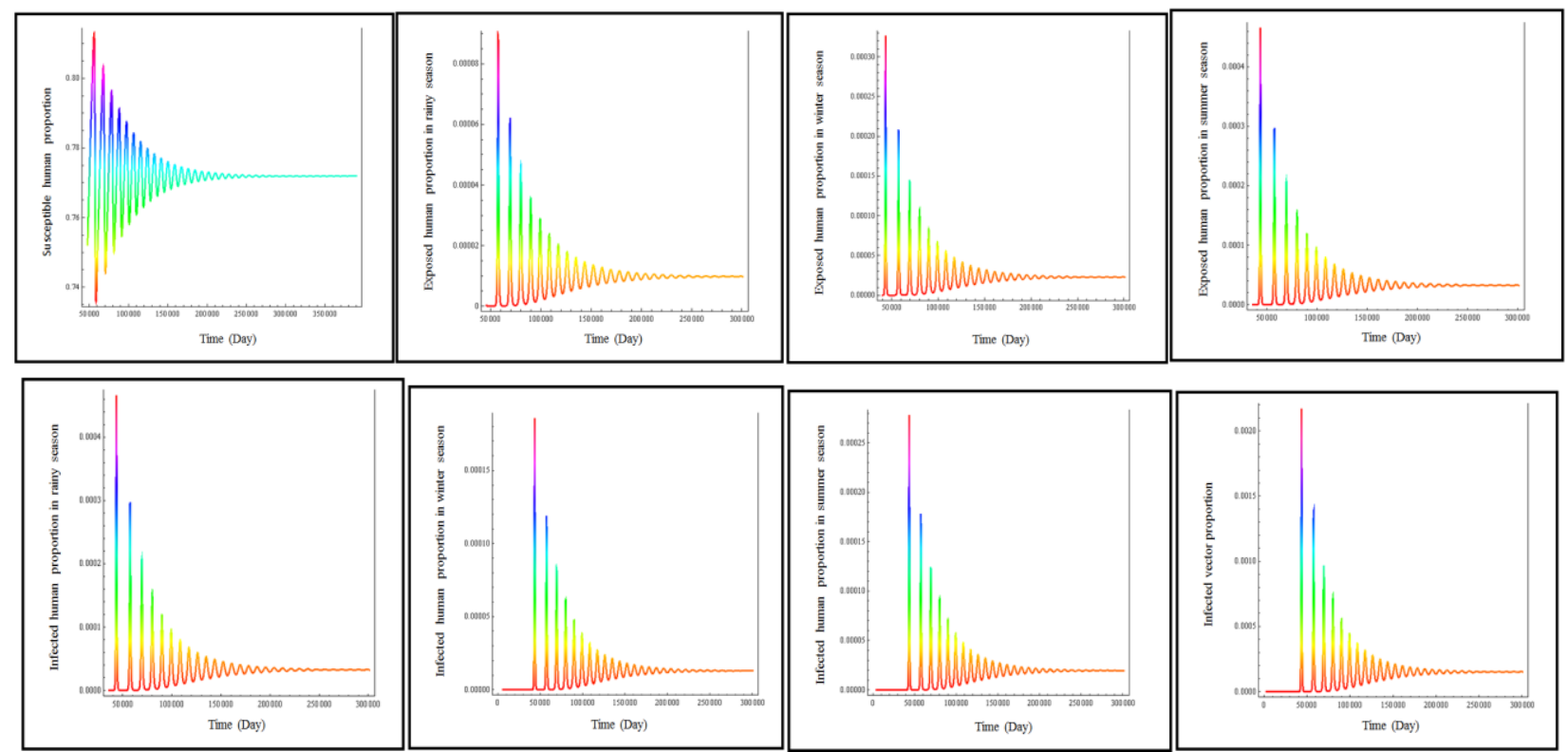

Fig. 3. Time series solutions of $S, E_{r}, E_{w}, E_{s}, I_{r}, I_{w}, I_{s}$ and $I_{v}$ where the parameters are given by $N_{t}=150,000, \tau_{d}=1 /(365 * 76.8), \tau_{v}=1 / 7, \beta_{r}=0.33, \beta_{w}=0.25, \beta_{s}=0.167, P_{r}=0.125, P_{w}=0.083$, $P_{s}=0.25, P_{v}=0.33, \alpha_{r}=0.33, \alpha_{w}=0.25, \alpha_{s}=0.142, \gamma_{r}=0.071, \gamma_{w}=0.10, \gamma_{s}=0.167, N_{v}=100,000$ and $R_{0}=1.2957$ The solutions converge to the endemic disease state $(0.771898,0.000009763,0.000006508,0.0000227785,0.0000325337,0.0000325337,0.0000195202$, 0.000151802 ). We can see that the solutions oscillate to the endemic disease state. 


\section{Conclusion}

In this study, the resulting model can indicate the presence in an endemic area for certain parameter values by using standard dynamical modeling method. We obtained two equilibrium states, namely the disease free equilibrium state and the endemic disease equilibrium state to each model.

Analytical results show that the number of each population human and mosquito population reaches a stable condition around the disease free equilibrium state with condition $R_{0}<1$ and stable around the endemic disease equilibrium state with condition $R_{0}>1$.

Fig 2 shows that a model with oscillates towards a stable value, converge to the disease free state.

Fig 3 shows the behaviors changes of solutions of the proportion of susceptible human population $(S)$, exposed human population in rainy season, winter season and summer season $\left(E_{r}, E_{w}, E_{s}\right)$, infectious human population in rainy season, winter season and summer season $\left(I_{r}, I_{w}, I_{s}\right)$, infectious mosquito population $\left(I_{v}\right)$ and the model solution oscillate to the disease endemic state[4,6,8,9].

\section{Appendix}

Appendixes, if needed, appear before the acknowledgment.

\section{Acknowledgment}

The author would like to thank Faculty of Science and Technology, Phuket Rajabhat University, King Mongkut's institute of Technology Ladkrabang and National Research Council of Thailand

\section{References}

[1] Bureau of Epidemiology. Department of Disease Control Ministry of Public Health. Retrieved from the website: http://www.boe.moph.go.th/index.php?nphss=nphss.

[2] World Health Organization. (1997). Dengue Haemorrhagic Fever: Diagnosis, Treatment, Prevention and Control. Geneva.

[3] Esteva, L., \& Vargas, C. (1998). Analysis of a dengue disease transmission model. Math BioSci, 15, 131-151.

[4] Sungchasit, R., Pongsumpun, P., \& Tang, I. M. (2013). Transmission model of dengue virus Aedes aegypti And Aedes albopictus. Far East Journal of Mathematical Sciences (FJMS), 83(1), 85-112.

[5] Pongsumpun, P., \& Tang, I. M. (2003). Transmission of dengue herrhagic fever in an age structured population. Mathematical and Computer Modeling, 37, 949-961.

[6] Kongnuy, R., \& Pongsumpun, P. (2010). Mathematical modeling for dengue transmission with the effect of season. Internation Journal of Biological and Medical Sciences, 5(2).

[7] Syafruddin, S. (2012). SEIR model for transmission of dengue fever in Selangor Malasia. International Journal of Modern Physics: Conference Series, 9, 380-389.

[8] Annelise, T., et al. (2013). A rainfall- and temperature-driven abundance model for Aedes albopictus populations. International Journal of Environmental Research and Public Health, 10, 1698-1719.

[9] Sungchasit, R., Pongsumpun, P., \& Tang, I. M. (2015). SIR transmission model of dengue virus taking into account two species of mosquitoes and an age structure in the human population. American Journal of Applied Sciences, 12(6), 426-443.

[10] Khalid, M. Mariam, S., Fareeha, S. K. (2015). Numerical solution of sir model of dengue fever. International Journal of Computer Applications, 118(21). 


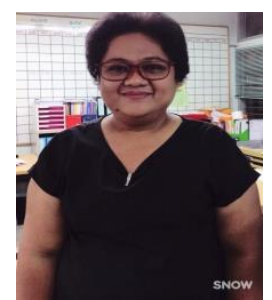

R. Sungchasit received her bachelor of education (graduate in mathematics) from Phuket Institute Rajahat, Thailand; the master of business administration (finance and banging) from Sripatum University, Thailand; the master of science program in applied statistics (statistics) from National Institute of Development Administration, Thailand; the doctor of philosophy (applied mathematics) from King Mongkut's Institute of Technology Landkrabang, Thailand. From 2007 till now, she is a lecturer in mathematics in Phuket Rajabhat University, Thailand. Her research interests are mathematical modelling.

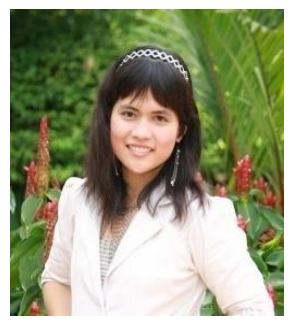

P. Pongsumpun received her bachelor of science in mathematics (second class honors) and the doctor of philosophy in mathematics (international programme) from Mahidol university, Thailand. From 2004 to 2012, she was an as assistant professor of mathematics. From 2012 till date, she is an associate professor of mathematics, thesis Ph.D. and M.Sc. advisors in King Mongkut's Institute of Technology Ladkrabang, Thailand. equations and numerical analysis. 